\title{
Erratum
}

\section{Defense Response to Pathogens Through Epigenetic Regulation in Rice}

Trung Viet Hoang, Kieu Thi Xuan Vo, Woo-Jong Hong, Ki-Hong Jung* and Jong-Seong Jeon*

In the original publication, a statement of equal contribution was not included. The text should read as: Trung Viet Hoang and Kieu Thi Xuan Vo contributed equally to this work. 